\title{
Creation of a Broad-Range and Highly Stereoselective D-Amino Acid Dehydrogenase for the One-Step Synthesis of D-Amino Acids
}

Kavitha Vedha-Peters, Manjula Gunawardana, J. David Rozzell, and Scott J. Novick*

BioCatalytics, Inc., 129 North Hill Avenue, Suite 103, Pasadena, CA 91106

\section{Supporting Information}

The following table contains the chromatography conditions used to analyzed the enantioselectivity of the mutant D-amino acid dehydrogenase (BC621). 


\begin{tabular}{|c|c|c|c|}
\hline D-Amino acid product & Column & $\begin{array}{l}\text { Retention } \\
\text { time (min) }\end{array}$ & Method \\
\hline $\begin{array}{l}\text { D-Alanine (D-2- } \\
\text { aminopropionate) }\end{array}$ & $\mathrm{T}$ & $\begin{array}{l}\text { L: } 8.3 \\
\text { D: } 9.6\end{array}$ & $\begin{array}{c}40 \% \text { methanol } \\
60 \% \text { water }(0.2 \% \text { TEAA, } \mathrm{pH} 3.8)\end{array}$ \\
\hline D-2-Aminobutyrate & $\mathrm{T}$ & $\begin{array}{l}\text { L: } 8.8 \\
\text { D: } 9.9\end{array}$ & $\begin{array}{l}20 \% \text { acetonitrile } \\
80 \% \text { water }(0.2 \% \text { TEAA, } \mathrm{pH} 3.8)\end{array}$ \\
\hline $\begin{array}{l}\text { D-Norvaline (D-2- } \\
\text { aminopentanoate) }\end{array}$ & $\mathrm{R}$ & $\begin{array}{l}\mathrm{L}: 7.5 \\
\mathrm{D}: 11.2\end{array}$ & $\begin{array}{c}20 \% \text { acetonitrile } \\
80 \% \text { water }(0.2 \% \text { TEAA, } \mathrm{pH} 3.8)\end{array}$ \\
\hline $\begin{array}{l}\text { D-Norleucine (D-2- } \\
\text { aminohexanoate) }\end{array}$ & $\mathrm{T}$ & $\begin{array}{l}\mathrm{L}: 8.7 \\
\mathrm{D}: 17.1\end{array}$ & $\begin{array}{l}40 \% \text { methanol } \\
60 \% \text { water }(0.2 \% \text { TEAA, } \mathrm{pH} 3.8)\end{array}$ \\
\hline D-2-Aminoheptanoate & $\mathrm{T}$ & $\begin{array}{l}\text { L: } 9.7 \\
\text { D: } 17.3\end{array}$ & $\begin{array}{l}40 \% \text { methanol } \\
60 \% \text { water }(0.2 \% \text { TEAA, } \mathrm{pH} 3.8)\end{array}$ \\
\hline D-2-Aminooctanoate & $\mathrm{T}$ & $\begin{array}{l}\text { L: } 11.5 \\
\text { D: } 21.3\end{array}$ & $\begin{array}{c}40 \% \text { methanol } \\
60 \% \text { water }(0.2 \% \text { TEAA, } \mathrm{pH} 3.8)\end{array}$ \\
\hline D-Valine & $\mathrm{T}$ & $\begin{array}{l}\text { L: } 9.37 \\
\text { D: } 10.5\end{array}$ & $\begin{array}{l}20 \% \text { acetonitrile } \\
80 \% \text { water }(0.2 \% \text { TEAA, } \mathrm{pH} 3.8)\end{array}$ \\
\hline D-Isoleucine & $\mathrm{T}$ & $\begin{array}{c}\mathrm{L}: 7.8 \\
\mathrm{D}: 12.5\end{array}$ & $\begin{array}{c}40 \% \text { methanol } \\
60 \% \text { water }(0.2 \% \text { TEAA, } \mathrm{pH} 3.8)\end{array}$ \\
\hline D-Leucine & $\mathrm{T}$ & $\begin{array}{l}\text { L: } 11.0 \\
\text { D: } 13.5\end{array}$ & $\begin{array}{l}20 \% \text { acetonitrile } \\
80 \% \text { water }(0.2 \% \text { TEAA, } \mathrm{pH} 3.8)\end{array}$ \\
\hline D-Cyclopentylglycine & $\mathrm{T}$ & $\begin{array}{l}\mathrm{L}: 9.3 \\
\mathrm{D}: 13.2\end{array}$ & $\begin{array}{l}40 \% \text { methanol } \\
60 \% \text { water }(0.2 \% \text { TEAA, } \mathrm{pH} 3.8)\end{array}$ \\
\hline D-Cyclohexylalanine & $\mathrm{T}$ & $\begin{array}{l}\text { L: } 7.9 \\
\text { D: } 8.9\end{array}$ & $\begin{array}{c}50 \% \text { methanol } \\
50 \% \text { water }(0.1 \% \text { each TEA, acetic acid })\end{array}$ \\
\hline D-Methionine & $\mathrm{T}$ & $\begin{array}{c}\text { L: } 8.2 \\
\text { D: } 17.0\end{array}$ & $\begin{array}{l}40 \% \text { methanol } \\
60 \% \text { water }(0.2 \% \text { TEAA, } \mathrm{pH} 3.8)\end{array}$ \\
\hline D-Phenylalanine & $\mathrm{T}$ & $\begin{array}{l}\text { L: } 5.6 \\
\text { D: } 7.3\end{array}$ & $\begin{array}{l}80 \% \text { methanol } \\
20 \% \text { water }(0.2 \% \text { TEAA, } \mathrm{pH} 3.8)\end{array}$ \\
\hline D-Tyrosine & $\mathrm{T}$ & $\begin{array}{l}\text { L: } 5.1 \\
\text { D: } 6.3\end{array}$ & $\begin{array}{l}80 \% \text { methanol } \\
20 \% \text { water }(0.2 \% \text { TEAA, } \mathrm{pH} 3.8)\end{array}$ \\
\hline D-4-Fluorophenylalanine & $\mathrm{T}$ & $\begin{array}{l}\text { L: } 5.4 \\
\text { D: } 6.7\end{array}$ & $\begin{array}{l}80 \% \text { methanol } \\
20 \% \text { water }(0.2 \% \text { TEAA, } \mathrm{pH} 3.8)\end{array}$ \\
\hline D-4-Chlorophenylalanine & $\mathrm{T}$ & $\begin{array}{l}\text { L: } 5.7 \\
\text { D: } 7.2\end{array}$ & $\begin{array}{c}80 \% \text { methanol } \\
20 \% \text { water }(0.2 \% \text { TEAA, } \mathrm{pH} 3.8)\end{array}$ \\
\hline
\end{tabular}

Notes:

- T: Chirobiotic T (Astec, Inc.), $250 \times 4.6 \mathrm{~mm}, 5 \mu \mathrm{m}$ particle size

- R: Chirobiotic R (Astec, Inc.), 250 x $4.6 \mathrm{~mm}, 5 \mu \mathrm{m}$ particle size

- The flow rate was $1 \mathrm{ml} / \mathrm{min}$ in all cases and all runs were performed isocratic conditions. 
- TEAA was made by adding triethylamine to water at the given concentration and adjusting to the given $\mathrm{pH}$ with acetic acid.

- All amino acid, except for the aromatic amino one, were labeled with FMOC for UV visualization at $265 \mathrm{~nm}$. The aromatic amino acids were observed directly at $254 \mathrm{~nm}$. 